\title{
Cilioretinal Sparing Central Retinal Artery Occlusion from Giant Cell Arteritis
}

\author{
Michelle Kasimov $^{a}$ Marko M. Popovic ${ }^{b}$ Jonathan A. Micielib, c, d \\ ${ }^{a}$ Faculty of Health, York University, Toronto, ON, Canada; ${ }^{b}$ Department of Ophthalmology \\ and Vision Sciences, University of Toronto, Toronto, ON, Canada; 'Kensington Vision and \\ Research Centre, Toronto, ON, Canada; ${ }^{d}$ Department of Ophthalmology, St. Michael's \\ Hospital/Unity Health Toronto, Toronto, ON, Canada
}

\author{
Keywords \\ Central retinal artery occlusion · Giant-cell arteritis · Vasculitis
}

\section{Abstract}

A 93-year-old woman presented with a 1-day history of sudden painless vision loss in her right eye with a background of new temporal headaches and jaw claudication. Dilated fundus examination of the right eye showed mild retinal whitening and edema throughout the right fundus with selective sparing in the distribution of the cilioretinal artery. A diagnosis of giantcell arteritis (GCA) was made and confirmed with temporal artery biopsy. Oral prednisone was initiated and her vision improved to 20/200 in the right eye at 3-month follow-up. GCA-related cilioretinal artery sparing central retinal artery occlusion is rare and may have mild improvement with promt treatment.

\section{Introduction}

Central retinal artery occlusion (CRAO) is usually a result of emboli to the central retinal artery circulation. Cilioretinal arteries exist in $15-30 \%$ of patients, and cilioretinal artery sparing CRAO can result in preserved or decreased central visual acuity [1]. This condition can rarely be seen in vasculitides such as giant-cell arteritis (GCA), for which 


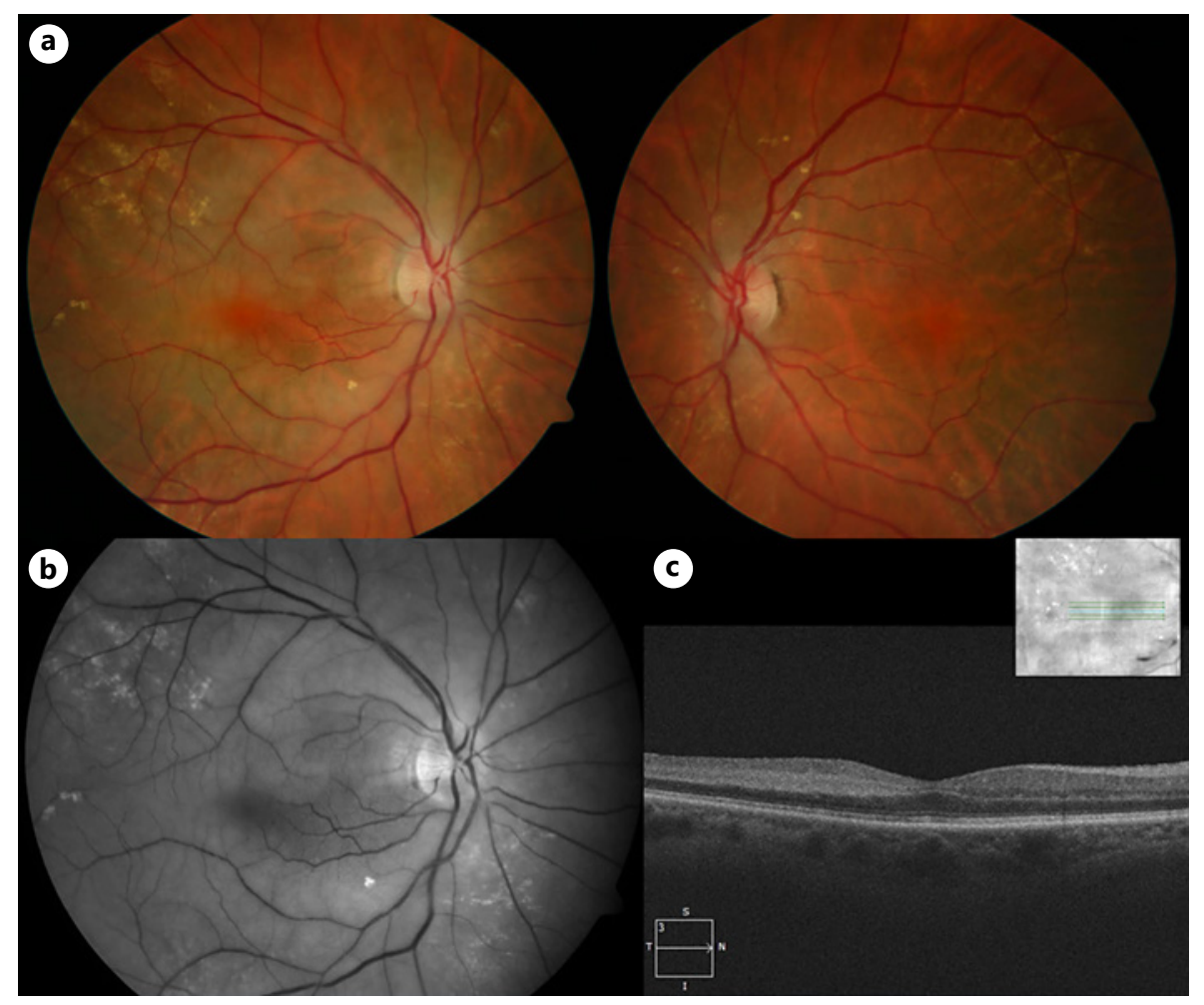

Fig. 1. a Color fundus photographs demonstrating diffuse retinal edema sparing the cilioretinal circulation in the right eye. b Red-free photographs highlighting the sparing of the cilioretinal circulation. c Optical coherence tomography of the macula demonstrating inner retinal edema temporal to the fovea and preserved retinal architecture nasal to the fovea.

only two previous case reports exist [2,3]. In previous cases, central visual acuity was severely reduced at presentation. Here, we present the third case of severe vision loss from GCA-related cilioretinal artery sparing CRAO.

\section{Case Description}

A 93-year-old woman presented with a 1-day history of sudden painless vision loss in her right eye. She also reported a 2-month history of new temporal headaches and a 1-week history of jaw claudication. She had a history of a cardiac arrhythmia, and her only medication was metoprolol. Visual acuity was hand motions in the right eye (OD) and 20/30 left eye (OS). Intraocular pressure was $7 \mathrm{~mm} \mathrm{Hg}$ OD and $10 \mathrm{~mm} \mathrm{Hg}$ OS. There was a right relative afferent pupillary defect. Anterior chamber examination of both eyes was unremarkable. Dilated fundus examination OD showed mild retinal whitening and edema throughout the right fundus with selective sparing in the distribution of the cilioretinal artery circulation (Fig. 1). There was an absence of cotton wool spots, no flameshaped or dot blot hemorrhages, and no optic-disc atrophy or edema. The left fundus was normal. She was diagnosed with a right cilioretinal artery sparing CRAO. Urgent bloodwork revealed thrombocytosis (platelets $443 \times 10^{9} / \mathrm{L}$ ), an erythrocyte sedimentation rate of $103 \mathrm{~mm} / \mathrm{h}$, and C-reactive protein of $159.6 \mathrm{mg} / \mathrm{L}$ (normal $<5 \mathrm{mg} / \mathrm{L}$ ). Given the high suspicion of GCA, she was started on prednisone $60 \mathrm{mg}$ (i.e., $1 \mathrm{mg} / \mathrm{kg}$ ) daily and underwent 
temporal artery biopsy, which revealed an inflammatory-cell infiltrate consisting of lymphocytes, macrophages, and multinucleated giant cells involving all three layers of the vessel. She was diagnosed with GCA and continued a prednisone taper. Her systemic symptoms improved within 1 month, and her visual acuity OD improved to 20/200 at 3 months of follow-up.

\section{Discussion}

In this case report, we describe a rare visual presentation of GCA, cilioretinal artery sparing CRAO. A cilioretinal artery is present in approximately $15-30 \%$ of eyes and originates from short posterior ciliary arteries or in rare cases, from the peripapillary choroid $[1,4]$. The cilioretinal artery supplies blood to the papillomacular bundle [5]. Typically, vision loss in GCA is a result of an ischemic optic neuropathy; however, in up to $10 \%$ of cases, patients lose vision due to CRAO [6, 7]. A study by Hayreh et al. [8] described a case series of GCA-related CRAO in 7 patients. In these cases, there was evidence of posterior ciliary artery circulatory abnormalities on intravenous fluorescein angiography. In cases with sparing of the cilioretinal artery circulation, the visual prognosis after GCA is less clear. Sane et al. [9] described the case of a 62-year-old woman with biopsy-confirmed GCA with episodes of vision loss in the right eye (visual acuity of 20/25 OD compared to 20/20 OS) that was found to have normal choroidal circulation but delayed filling of the retinal arterioles. This case affected the central retinal artery as it came off the ophthalmic artery, and the posterior ciliary circulation was relatively spared [9].

Cilioretinal artery sparing CRAO resulting from GCA is very rare and has been reported before in two previous publications. A report by Cohen and Rizzo [2] described the case of a 79-year-old woman who developed headaches, jaw claudication, and vision loss OS to hand motions. A temporal artery biopsy confirmed GCA. The final visual acuity and treatment received were not reported [2]. Connolly et al. [3] presented the case of a 79-year-old woman with a history of right exotropia and right amblyopia with sudden vision loss OS and an erythrocyte sedimentation rate of $140 \mathrm{~mm} / \mathrm{h}$. Visual acuity at presentation was counting fingers OD and light perception OS. Fundoscopy was normal OD but showed retinal whitening consistent with a cilioretinal artery sparing CRAO in the left eye. She was prescribed an initial dose of intravenous corticosteroids followed by oral corticosteroids, and temporal artery biopsy confirmed GCA. No follow-up data were provided [3]. In all reported cases including ours, the presenting visual acuity in GCA-related cilioretinal artery sparing CRAO was poor. This may be primarily attributable to additional involvement of the choroidal circulation, which is typically affected in these cases. Our case is the first that provides follow-up data on visual prognosis and suggests that with appropriate treatment, an improvement in vision is possible.

Hayreh [1] divided CRAO into four different clinical entities: nonarteritic CRAO, nonarteritic CRAO with cilioretinal artery sparing, arteritic CRAO associated with GCA, and transient nonarteritic CRAO. Our case report presents a distinct subtype of the third category, namely arteritic cilioretinal artery sparing CRAO associated with GCA [1]. A study by Liu et al. [10] showed that the sparing of cilioretinal artery in CRAO is important in limiting ischemia to the macula and improving the final visual prognosis. Similar prognostic relationships have been described in other studies $[5,11]$. For instance, a study by Kim et al. [5] studied 90 eyes with CRAO, 16 of which were found to have sparing of the cilioretinal artery. The proportion with a posttreatment best-corrected visual acuity worse than $20 / 200$ was $81.3 \%$ in the cilioretinal artery sparing group and $97.3 \%$ if the cilioretinal artery was involved ( $p=0.01$ ) [5]. However, studies such as these have focused on nonar-

\section{Karger'}


Kasimov et al.: Cilioretinal Sparing CRAO in GCA

teritic CRAO, and it is uncertain whether these observations hold true in patients with GCA. Hayreh et al. [12] found that in patients with vision loss due to GCA, either through CRAO or other GCA-related mechanisms, only $4 \%$ of eyes out of 114 studied improved vision after steroid treatment. Although the extent of improvement is rare, the study did not mention any patients with cilioretinal artery sparing CRAO, and thus, it is unknown whether the finding is applicable to patients like the one in our case. Furthermore, it is unclear whether steroid dosing and the route of administration affect final visual acuity in arteritic CRAO with sparing of the cilioretinal artery.

Our case has the advantage of having fundus photography and optical coherence tomography at presentation. However, it was limited in that similar to previous cilioretinal artery occlusions in GCA, intravenous fluorescein angiography was not available. Our patient was elderly and had a hard time positioning for this test, and therefore, fluorescein angiography was not possible.

\section{Conclusion}

Our report presents the third documented case of a patient with cilioretinal artery sparing CRAO as a result of GCA and the only case with follow-up data. Sparing of the cilioretinal artery has been shown to improve the prognosis of nonarteritic CRAO, but more research is needed to explore how it affects the prognosis of patients in the context of GCA. Our cause suggests there may be mild improvement with prompt treatment.

\section{Statement of Ethics}

Official ethics approval was not required in accordance with the St. Michael's Hospital protocol. Written informed consent was obtained from the patient for publication of this case report and any accompanying images.

\section{Conflict of Interest Statement}

The authors have no conflicts of interest to declare.

\section{Funding Sources}

No funding sources were used for the preparation of data or the manuscript.

\section{Author Contributions}

Michelle Kasimov and Marko M. Popovic conducted the literature review and wrote the manuscript. Jonathan A. Micieli is the principal investigator who assisted with data analysis, interpretation, and critical review of the manuscript.

\section{Karger'k}


Kasimov et al.: Cilioretinal Sparing CRAO in GCA

\section{Data Availability Statement}

The patient's medical information is stored in their medical record. No other datasets were used.

\section{References}

1 Hayreh SS. Acute retinal arterial occlusive disorders. Prog Retin Eye Res. 2011 Sep;30(5):359-94.

2 Cohen AB, Rizzo JF 3rd. Teaching NeuroImages: central retinal artery occlusion with cilioretinal artery sparing in giant cell arteritis. Neurology. 2010 Aug 3;75(5):e21-2.

3 Connolly BP, Krishnan A, Shah GK, Whelan J, Brown GC, Eagle RC Jr, et al. Characteristics of patients presenting with central retinal artery occlusion with and without giant cell arteritis. Can J Ophthalmol. 2000 Dec;35(7): 379-84.

4 Brown GC, Shields JA. Cilioretinal arteries and retinal arterial occlusion. Arch Ophthalmol. 1979 Jan; 97(1): 84-92.

5 Kim YH, Park KH, Woo SJ. Clinical manifestations and visual prognosis of cilioretinal artery sparing central retinal artery occlusion. Korean J Ophthalmol. 2020 Feb;34(1):27-34.

6 Appen RE, Wray SH, Cogan DG. Central retinal artery occlusion. Am J Ophthalmol. 1975 Mar;79(3):374-81.

7 Eagling EM, Sanders MD, Miller SJ. Ischaemic papillopathy. Clinical and fluorescein aniographic review of forty cases. Br J Ophthalmol. 1974 Dec;58(12):990-1008.

8 Hayreh SS, Podhajsky PA, Zimmerman B. Ocular manifestations of giant cell arteritis. Am J Ophthalmol. 1998 Apr;125(4):509-20.

9 Sane M, Selvadurai A, Reidy J, Higgs D, Gonzalez-Fernandez F, Lincoff N. Transient visual loss due to reversible "pending" central retinal artery occlusion in occult giant cell arteritis. Eye. 2014 Nov;28(11):1387-90.

10 Liu L, Liu LM, Chen L. Incidence of cilioretinal arteries in Chinese Han population. Int J Ophthalmol. 2011;4(3): 323-5.

11 Hayreh SS, Zimmerman MB. Central retinal artery occlusion: visual outcome. Am J Ophthalmol. 2005 Sep; 140(3):376-91.

12 Hayreh SS, Zimmerman B, Kardon RH. Visual improvement with corticosteroid therapy in giant cell arteritis. Report of a large study and review of literature. Acta Ophthalmol Scand. 2002 Aug;80(4):355-67. 